\title{
On equivalence of the second order linear differential operators, acting in vector bundles
}

\author{
Valentin Lychagin ${ }^{\mathrm{a}, *}$ \\ ${ }^{a}$ V.A. Trapeznikov Institute of Control Sciences, Russian Academy of Sciences, 65 \\ Profsoyuznaya Str., 117997 Moscow, Russia
}

\begin{abstract}
The equivalence problem for linear differential operators of the second order, acting in vector bundles, is discussed. The field of rational invariants of symbols is described and connections, naturally associated with differential operators, are found. These geometrical structures are used to solve the problems of local as well as global equivalence of differential operators.
\end{abstract}

Keywords: differential operator, differential invariant, equivalence problem, vector bundle

2010 MSC: 58J70, 53C05, 35A30, 35G05

\section{Introduction}

This paper is the last in the series of papers devoted to invariants, geometrical structures and equivalence of linear differential operators, acting in vector bundles $([\underline{5}],[4],[6])$. Here we study linear differential operators of the second order, acting in sections of a vector bundle $\pi: E(\pi) \rightarrow M$, where $\operatorname{dim} M=n \geq 2, \operatorname{dim} \pi=m \geq 2$.

Our approach is based on the following main steps. First of all we study geometrical structures defined by symbols of differential operators and use them to define invariants and orbits of the symbols with respect to groups, induced by automorphisms of bundles. The second step is extremely important for us and consist of finding connections, naturally associated with differential operators. Thus in the given case, in general, the symbol of the differential operators defines a pseudo Riemannian structure on the base manifold and the condition of the triviality of the subsymbol leads us to uniquely defined connection in the bundle. This connection is naturally related to the differential operator and gives us the quantization, or splitting of differential operator into the sum of the symbol and an endomorphism of the bundle. After this, the splitting and the Artin-Procesi theorem $([1],[3])$ allow us to find invariants of operators. The last step of the

\footnotetext{
${ }^{*}$ Corresponding author

Email address: valentin.lychagin@uit.no (Valentin Lychagin)
} 
approach (similar to the one used in [5], [4], 6] ) consist of in using the invariants to construct the natural coordinates and local models of differential operators. This gives us local and global description of differential operators under some natural conditions of regularity on the operator.

\section{Differential operators}

\subsection{Preliminaries}

The notations we use in this paper are similar to the ones that were used in the papers [4] and [6] .

Let $M$ be an $n$-dimensional manifold and let $\pi: E(\pi) \rightarrow M$ be a vector bundle.

We denote by $\tau: T M \rightarrow M$ and $\tau^{*}: T^{*} M \rightarrow M$ the tangent and respectively cotangent bundles over the manifold $M$, and by $1: \mathbb{R} \times M \rightarrow M$ we denote the trivial linear bundle .

The symmetric and exterior powers of a vector bundle $\pi: E(\pi) \rightarrow M$ will be denoted by $\mathbf{S}^{k}(\pi)$ and $\boldsymbol{\Lambda}^{k}(\pi)$.

The module of smooth sections of bundle $\pi$ we denote by $C^{\infty}(\pi)$, and for the cases tangent, cotangent and the trivial bundles we will use the following notations: $\Sigma_{k}(M)=C^{\infty}\left(\mathbf{S}^{k}(\tau)\right)-$ is the module of symmetric $k$-vectors and $\Sigma^{k}(M)=C^{\infty}\left(\mathbf{S}^{k}\left(\tau^{*}\right)\right)$ - is the module of symmetric $k$-forms, $\Omega_{k}(M)=$ $C^{\infty}\left(\Lambda^{k}(\tau)\right)$ - is the module of skew-symmetric $k$-vectors and $\Omega^{k}(M)=C^{\infty}\left(\Lambda^{k}\left(\tau^{*}\right)\right)$ -is the module of exterior $k$-forms, $C^{\infty}(\mathbf{1})=C^{\infty}(M)$.

Let $\operatorname{Diff}_{k}(\pi, \pi)$ be the module of linear differential operators of order $k$ acting in the sections of the bundle $\pi$. These modules are connected by the exact sequences

$$
\mathbf{0} \rightarrow \operatorname{Diff}_{k-1}(\pi, \pi) \rightarrow \operatorname{Diff}_{k}(\pi, \pi) \stackrel{\mathrm{smbl}_{k}}{\rightarrow} \operatorname{End}(\pi) \otimes \Sigma_{k}(M) \rightarrow \mathbf{0},
$$

where End $(\pi)=\operatorname{Diff}_{0}(\pi, \pi)$ is the module of endomorphisms of $C^{\infty}(\pi)$, the mapping smbl assigns to differential operator $\Delta$ its $\operatorname{symbol} \operatorname{smbl}_{k}(\Delta)$ and the tensor product is taking over $C^{\infty}(M)$.

If we consider $\operatorname{smbl}_{k}(\Delta)$ as a linear operator $\operatorname{smbl}_{k}(\Delta): \Sigma^{k}(M) \rightarrow \operatorname{End}(\pi)$, then

$$
\operatorname{smbl}_{k}(\Delta)\left(d f^{k}\right)=\frac{1}{k !} \delta_{f}^{k}(\Delta) \in \operatorname{End}(\pi),
$$

where $f \in C^{\infty}(M), \delta_{f}: \operatorname{Diff}_{i}(\pi, \pi) \rightarrow \operatorname{Diff}_{i-1}(\pi, \pi)$, is the commutator mapping $\delta_{f}(\square)=f \circ \square-\square \circ f$, and $d f^{k}=d f \cdots \cdot d f \in \Sigma^{k}(M)$ is the $k$-th symmetric power of the differential 1-form $d f \in \Sigma^{1}(M)$.

\subsection{Pseudogroup actions}

As before $([4])$, we consider two pseudogroups: $\mathcal{G}(M)-$ the pseudogroup of local diffeomorphisms of manifold $M$, and $\operatorname{Aut}(\pi)$ - the pseudogroups of local automorphisms of vector bundle $\pi$ over $M$. 
The following sequence of pseudogroup morphisms is exact

$$
1 \rightarrow \mathrm{GL}(\pi) \rightarrow \operatorname{Aut}(\pi) \rightarrow \mathcal{G}(M) \rightarrow 1,
$$

where $\operatorname{GL}(\pi) \subset \operatorname{Aut}(\pi)$ is the pseudogroup of automorphisms that are identity on $M$.

We will consider the natural actions of these pseudogroups on sections of the bundles and on operators. Namely, let $\widetilde{\phi}$ be a local automorphism, $\widetilde{\phi} \in \operatorname{Aut}(\pi)$, covering a local diffeomorphism $\phi \in \mathcal{G}(M)$.

We define action of $\widetilde{\phi}$ on sections $s \in C^{\infty}(\pi)$ as

$$
\widetilde{\phi}_{*}: s \longmapsto \widetilde{\phi} \circ s \circ \phi^{-1},
$$

and

$$
\widetilde{\phi}_{*}: \Delta \longmapsto \widetilde{\phi}_{*} \circ \Delta \circ \widetilde{\phi}_{*}^{-1},
$$

for differential operators.

\section{Quantizations, symbols and subsymbols}

Let $\Sigma=\oplus_{k \geq 0} \Sigma^{k}(M)$ be the graded algebra of symmetric differential forms and let $\Sigma(\pi)=C^{\infty}(\pi) \otimes \Sigma$ be the graded $\Sigma$-module of symmetric differential forms with values in the bundle $\pi$.

Let $\nabla$ be a connection in the vector bundle $\pi$ and let $\nabla_{c}$ be a connection in the cotangent bundle $\tau^{*}$.

Then their covariant differentials

$$
d_{\nabla_{c}}: \Omega^{1}(M) \rightarrow \Omega^{1}(M) \otimes \Omega^{1}(M),
$$

and

$$
d_{\nabla}: C^{\infty}(\pi) \rightarrow C^{\infty}(\pi) \otimes \Omega^{1}(M)
$$

define derivation

$$
d_{\nabla_{c}}: \Sigma^{\cdot} \rightarrow \Sigma^{++1}
$$

of degree one in graded algebra $\Sigma$ and derivation

$$
d_{\nabla}: \Sigma(\pi) \rightarrow \Sigma^{\cdot+1}(\pi)
$$

of degree one in graded $\Sigma$-module $\Sigma \cdot(\pi)$ respectively.

Indeed, derivations are completely defined by their actions on generators.

In our case, we get

$$
\begin{aligned}
& d_{\nabla_{c}}=d: C^{\infty}(M) \rightarrow \Omega^{1}(M)=\Sigma^{1}, \\
& d_{\nabla_{c}}: \Omega^{1}(M)=\Sigma^{1} \stackrel{d}{\rightarrow} \Omega^{1}(M) \otimes \Omega^{1}(M) \stackrel{\text { Sym }}{\rightarrow} \Sigma^{2},
\end{aligned}
$$

where Sym denotes the symmetrization, and define $d_{\nabla}$ as a derivation over $d_{\nabla_{c}}$ such that

$$
d_{\nabla}: C^{\infty}(\pi) \rightarrow C^{\infty}(\pi) \otimes \Sigma^{1} .
$$


is the covariant differential.

Let now $\sigma \in \operatorname{End}(\pi) \otimes \Sigma_{k}$ be a symbol. We define the differential operator $\widehat{\sigma} \in \operatorname{Diff}_{k}(\pi, \pi)$ as follows:

$$
\widehat{\sigma}(s) \stackrel{\text { def }}{=} \frac{1}{k !}\left\langle\sigma,\left(d_{\nabla}\right)^{k}(s)\right\rangle,
$$

where $s \in C^{\infty}(\pi),\left(d_{\nabla}\right)^{k}(s) \in C^{\infty}(\pi) \otimes \Sigma^{k}$,and $\langle\cdot, \cdot\rangle$ is the natural pairing

$$
\text { End }(\pi) \otimes \Sigma_{k} \otimes C^{\infty}(\pi) \otimes \Sigma^{k} \rightarrow C^{\infty}(\pi) .
$$

Remark that the value of the symbol of the derivation $d_{\nabla}$ on a covector $\theta$ equals the symmetric product by $\theta$ in the module $\Sigma(\pi)$ and because the symbol of a composition of operators equals the composition of symbols we get that the symbol of the operator $\widehat{\sigma}$ equals $\sigma$.

We call this operator $\widehat{\sigma}$ the quantization of symbol $\sigma$ and write $\widehat{\sigma}=Q_{\nabla}(\sigma)$.

By the construction morphism $Q_{\nabla}:$ End $(\pi) \otimes \Sigma_{k} \rightarrow \operatorname{Diff}_{k}(\pi, \pi)$ splits sequence (1).

Let now $A \in \operatorname{Diff}_{k}(\pi, \pi)$ be a differential operator and $\sigma_{k}(A) \in \operatorname{End}(\pi) \otimes \Sigma_{k}$ be its symbol. Then operator

$$
A-Q_{\nabla}\left(\sigma_{k}(A)\right)
$$

has order $(k-1)$, and let $\sigma_{k-1}(A, \nabla) \in$ End $(\pi) \otimes \Sigma_{k-1}$ be its symbol.

Then operator $A-Q_{\nabla}\left(\sigma_{k}(A)\right)-Q_{\nabla}\left(\sigma_{k-1}(A, \nabla)\right)$ has order $(k-2)$. Repeating this process we get subsymbols $\sigma_{i}(A, \nabla) \in \operatorname{End}(\pi) \otimes \Sigma_{i}, 0 \leq i \leq k-1$, such that

$$
A=Q_{\nabla}(\sigma(A, \nabla)),
$$

where

$$
\sigma(A, \nabla)=\oplus_{0 \leq i \leq k} \sigma_{i}(A, \nabla)
$$

is a total symbol of the operator, and $Q_{\nabla}(\sigma(A, \nabla))=\sum_{i} Q_{\nabla}\left(\sigma_{i}(A, \nabla)\right)$.

Theorem 1. Let $\widetilde{\nabla}$ be a connection in the bundle $\pi$ and let $\widetilde{\nabla}=\nabla+\alpha$ be another connection, where $\alpha \in \operatorname{End}(\pi) \otimes \Sigma^{1}(M), \widetilde{\nabla}_{X}=\nabla_{X}+\alpha(X)$.

Then

$$
\sigma_{k-1}(A, \nabla)=\sigma_{k-1}(A, \widetilde{\nabla})+\left\langle\sigma_{k}, \alpha\right\rangle,
$$

where $\left\langle\sigma_{k}, \alpha\right\rangle \in \operatorname{End}(\pi) \otimes \Sigma_{k-1}$ is the natural pairing $\operatorname{End}(\pi) \otimes \Sigma_{k} \times \operatorname{End}(\pi) \otimes$ $\Sigma^{1}(M) \rightarrow \operatorname{End}(\pi) \otimes \Sigma_{k-1}$.

Proof. Remark that $\delta_{f}\left(d_{\nabla}\right)=d f$ and

$$
\delta_{f}^{i}\left(d_{\nabla}^{k}\right)=k(k-1) \cdots(k-i+1) d f^{i} \cdot d_{\nabla}^{k-i} .
$$

Therefore,

$$
\delta_{f}^{k-1}\left(d_{\nabla}^{k}\right)=k ! d f^{k-1} \cdot d_{\nabla},
$$


and

$$
\delta_{f}^{k-1}\left(Q_{\nabla}\left(\sigma_{k}\right)\right)(s)=\left\langle\sigma_{k}, d f^{k-1} \cdot d_{\nabla}(s)\right\rangle
$$

Applying this formula to connection $\nabla+\alpha$ we get the statement of the theorem:

$$
\begin{aligned}
& \left.d f^{k-1}\right\rfloor\left(\sigma_{k-1}(A, \nabla)-\sigma_{k-1}(A, \widetilde{\nabla})\right)=\delta_{f}^{k-1}\left(Q_{\widetilde{\nabla}}\left(\sigma_{k}\right)-Q_{\nabla}\left(\sigma_{k}\right)\right)= \\
& \left.\left\langle\sigma_{k}, d f^{k-1}\left(d_{\nabla+\alpha}-d_{\nabla}\right)\right\rangle=\left\langle\sigma_{k}, d f^{k-1} \cdot \alpha\right\rangle=d f^{k-1}\right\rfloor\left\langle\sigma_{k}, \alpha\right\rangle,
\end{aligned}
$$

where $\rfloor$ stands for the inner product. We apply now this result to operators of the order two.

At first, we say that the symbol $\sigma_{2} \in \operatorname{End}(\pi) \otimes \Sigma_{2}$ of an operator $A \in$ $\operatorname{Diff}_{2}(\pi, \pi)$ is regular (later we'll extend this notion) if the following morphism

$$
\begin{array}{ll}
\widehat{\sigma_{2}}: & \operatorname{End}(\pi) \otimes \Sigma^{1} \rightarrow \operatorname{End}(\pi) \otimes \Sigma_{1}, \\
\widehat{\sigma_{2}}: & \alpha \in \operatorname{End}(\pi) \otimes \Sigma^{1} \longmapsto\left\langle\sigma_{2}, \alpha\right\rangle \in \operatorname{End}(\pi) \otimes \Sigma_{1},
\end{array}
$$

is an isomorphism.

The next result (cf. [5]) is the direct corollary of the above theorem.

Theorem 2. Let $A \in \operatorname{Diff}_{2}(\pi, \pi)$ be an operator with regular symbol. Then, for any connection $\nabla_{c}$ in the cotangent bundle there is and unique connection $\nabla$ in the bundle $\pi$, such that $\sigma_{1}(A, \nabla)=0$.

\section{Symbol classification}

In this section we consider symbols of the second order operators at a point as tensors of the form $\sigma \in$ End $(E) \otimes S^{2} T$, where $E$ and $T$ are vector spaces of dimensions $m=\operatorname{dim} E \geq 2$ and $n=\operatorname{dim} T \geq 2$, and study their $G=$ GL $(E) \times$ GL $(T)$-orbits (cf. [4]). All constructions in this section have pure algebraic nature and can be used over the field $\mathbb{R}$ as well as the field $\mathbb{C}$.

We denote by $\sigma_{\theta} \in \operatorname{End}(E)$ the value of the symbol on a tensor $\theta \in S^{2} T^{*}$, and let

$$
\begin{aligned}
& g_{\sigma}=(\operatorname{Tr} \otimes \mathrm{id}) \sigma \in S^{2} T, \\
& \left\langle g_{\sigma}, \theta\right\rangle=\operatorname{Tr}\left(\sigma_{\theta}\right),
\end{aligned}
$$

be the quadratic form, associated with the symbol.

Similar to [4] we introduce Artin-Procesi tensors

$$
A_{\sigma, I}\left(\theta_{1} \otimes \cdots \otimes \theta_{k}\right)=\operatorname{Tr}\left(\sigma_{\theta_{i_{1}}} \cdots \sigma_{\theta_{i_{k}}}\right),
$$

where $I=\left(i_{1}, \ldots, i_{k}\right) \in S_{k}$ is a permutation of $k$-letters, and $\theta_{i} \in S^{2} T^{*}$.

Thus, tensors $A_{\sigma, I} \in\left(\left(S^{2} T^{*}\right)^{\otimes k}\right)^{*}=\left(S^{2} T\right)^{\otimes k}$ are $G$-invariants and $A_{\sigma, I}=$ $A_{\sigma, J}$, when permutation $J \in S_{k}$ is obtained from $I$ by a cycle permutation. 
Besides Artin-Procesi quadric $g_{\sigma}$, the following tensors $h_{2}=A_{\sigma,(1,2)} \in$ $\left(S^{2} T\right)^{\otimes 2}, h_{3}=A_{\sigma,(1,2,3)} \in\left(S^{2} T\right)^{\otimes 3}$,

$$
\begin{aligned}
h_{2}\left(\theta_{1}, \theta_{2}\right) & =\operatorname{Tr}\left(\sigma_{\theta_{1}} \sigma_{\theta_{2}}\right), \\
h_{3}\left(\theta_{1}, \theta_{2}, \theta_{3}\right) & =\operatorname{Tr}\left(\sigma_{\theta_{1}} \sigma_{\theta_{2}} \sigma_{\theta_{3}}\right)
\end{aligned}
$$

will be extremely important for us.

In what follows we will use symbols in general position, or general symbols, i.e symbols where the following regularity conditions, together with (4), hold (cf. 4]).

Namely, we require the following:

1. First of all we require that the quadric $g_{\sigma} \in S^{2} T$ on $T^{*}$ is nondegenerate. Denote by $g_{\sigma}^{-1} \in S^{2} T^{*}$ the inverse quadric on $T$ and let $\left.g_{\sigma}^{(1)}=g_{\sigma}^{-1}\right\rfloor h_{2} \in$ $\left.\left.S^{2} T, g_{\sigma}^{(2)}=g_{\sigma}^{-1}\right\rfloor g_{\sigma}^{-1}\right\rfloor h_{3} \in S^{2} T$ be another quadratic forms on $T^{*}$.

2. Denote by $\widehat{g}_{\sigma}^{(1)}: T^{*} \rightarrow T^{*}$ the linear operator, associated with the pair of quadratic forms $\left(g_{\sigma}, g_{\sigma}^{(1)}\right)$ on $T^{*}$. We require that $\widehat{g}_{\sigma}^{(1)}$ as well as its symmetric power $S=S^{2}\left(\widehat{g}_{\sigma}^{(1)}\right): S^{2} T^{*} \rightarrow S^{2} T^{*}$ are are isomorphisms with distinct eigenvalues. Moreover, we require that quadrics

$$
g_{\sigma}^{(2)}, S^{*}\left(g_{\sigma}^{(2)}\right), \ldots \ldots,\left(S^{*}\right)^{N}\left(g_{\sigma}^{(2)}\right)
$$

where $N=\operatorname{dim} S^{2} T^{*}-1$ are linear independent.

3. The eigenvector basis of the operator $\widehat{g}_{\sigma}^{(1)}$ in $T^{*}$ (possibly over $\mathbb{C}$ ) we denote by $e^{*}(\sigma)$ and the dual basis by $e(\sigma)$. We will require, in addition, that the values $g_{\sigma}\left(e_{i}^{*}, e_{i}^{*}\right) \neq 0$, and eigenvectors are normed in such a way that, $g_{\sigma}\left(e_{i}^{*}, e_{i}^{*}\right)= \pm 1$.

4. The last regularity condition requires that almost all operators $\sigma_{\theta}$ has distinct eigenvalues and the commutator map $\sigma_{\theta_{1}} \times \sigma_{\theta_{2}} \longmapsto\left[\sigma_{\theta_{1}}, \sigma_{\theta_{2}}\right]$ for these operators is nontrivial.

Denote by $\mathbb{F}$ the splitting field of the characteristic polynomial $\operatorname{det}\left(\widehat{g}_{\sigma}^{(1)}-\lambda\right)$ over field $\mathbb{F}_{0}$ of the rational functions on the symbol space End $(E) \otimes S^{2} T$.

Let $E_{\mathbb{F}}=\mathbb{F} \otimes_{\mathbb{R}} E, T_{\mathbb{F}}=\mathbb{F} \otimes_{\mathbb{R}} T$ and let $e^{*}(\sigma)$ let be an eigenvector basis in $T_{\mathbb{F}}^{*}$ of the operator $\widehat{g}_{\sigma}^{(1)}$ and $e(\sigma)$ be the dual basis in $T_{\mathbb{F}}$.. Denote by $\lambda(\sigma)$ the corresponding eigenvalues.

Let now

$$
\sigma=\sum_{i, j} \sigma_{i j} \otimes e_{i} \otimes e_{j}
$$

be decomposition of the symbol in the eigenvector basis, where operators $\sigma_{i j} \in$ End $E_{\mathbb{F}}$.

Define operators

$$
R(\sigma)=\sum_{i, j} \lambda_{i}^{l} \lambda_{j}^{l} g_{\sigma}^{(2)}\left(e_{i}^{*}, e_{j}^{*}\right) \sigma_{i j} \in \operatorname{End} E_{\mathbb{F}},
$$


for $l=0,1, \ldots$

Remark that these operators are invariants with respect to transpositions and therefore all $S_{n}$-permutations of eigenvalues and transformations of the sign change: $e_{i} \rightarrow \pm e_{i}$.

Therefore, $R_{l}(\sigma) \in$ End $E_{\mathbb{F}_{0}}$, are operators with rational coefficients.

Lemma 3. Assume that generality conditions $(1-4)$ hold. Then operators $R_{l}(\sigma), l=0,1, \ldots,(n+2)(n-1) / 2$, define the symbol $\sigma$ uniquely.

Proof. Denote by $\widetilde{\lambda}_{l}$ the following quadratic forms $\widetilde{\lambda}_{l}=\sum_{i, j} \lambda_{i}^{l} \lambda_{j}^{l} g_{\sigma}^{(2)}\left(e_{i}^{*}, e_{j}^{*}\right) e_{i}^{*} \otimes$ $e_{j}^{*} \in S^{2}\left(T_{\mathbb{F}}^{*}\right)$, where $l=0,1, \ldots$

Then $\widetilde{\lambda}_{0}=g_{\sigma}^{(2)}$ and $\widetilde{\lambda}_{l+1}=S\left(\widetilde{\lambda}_{l}\right)$. Therefore, forms $\widetilde{\lambda}_{l}, l=0,1, \ldots .,(n+2)(n-1) / 2$ give a basis in $S^{2}\left(T_{\mathbb{F}}^{*}\right)$ and, because of $R_{l}(\sigma)=\left\langle\sigma, \widetilde{\lambda}_{l}\right\rangle$, with respect to the natural pairing End $E_{\mathbb{F}} \otimes S^{2}\left(T_{\mathbb{F}}\right) \times S^{2}\left(T_{\mathbb{F}}^{*}\right) \rightarrow$ End $E_{\mathbb{F}}$, operators $R_{l}(\sigma)$ define the symbol.

This lemma shows that $G$-equivalence of symbols is equivalent to GL $\left(E_{\mathbb{F}_{0}}\right)$ equivalence of the sets of operators $\left\{R_{l}(\sigma)\right\}$, and therefore, due to the ArtinProcesi theorem, is completely described by the Artin-Procesi invariants

$$
A_{I}(\sigma)=\operatorname{Tr}\left(R_{i_{1}}(\sigma) \cdots R_{i_{s}}(\sigma)\right)
$$

where $I=\left(i_{1}, \ldots, i_{s}\right), 0 \leq i_{j} \leq(n+1)(n-2) / 2$.

The Artin-Procesi theorem ([3]) together with the Rosenlicht theorem ([7]) give us the following result.

Theorem 4. The field of rational $G$-invariants of symbols $\sigma \in$ End $E \otimes S^{2}(T)$ is generated by the invariants (5). This field separates regular orbits.

Let's now $\sigma \in$ End $E \otimes S^{2}(T)$ be a symbol in general position. Then, due to condition (3) the stationary Lie algebra of the orbit $G \sigma$ is one dimensional, and therefore

$$
\nu=\operatorname{codim}(G \sigma)=m^{2} \frac{(n+2)(n-1)}{2}-n^{2}+1 .
$$

Definition 1. In addition to the above notion of regularity we will say that a general symbol is regular if there are $\nu$ rational $G$-invariants $f_{1}, \ldots, f_{\nu}$ that define the orbit $G \sigma$, their differentials are independent at the points of the orbit, and condition (4) holds.

\section{Associated connections and invariants of operators}

Let $\Delta \in \operatorname{Diff}_{2}(\pi, \pi)$ be a differential operator with regular symbol $\sigma=$ $\operatorname{smbl}_{2}(\Delta) \in \operatorname{End}(\pi) \otimes \Sigma_{2}$. Then the quadric $g_{\sigma} \in \Sigma_{2}$ is nondegenerate and we denote by $\nabla_{c}$ the associated Levi-Civita connection. Taking this connection in 
Theorem 2 , we get a connection $\nabla=\nabla^{\Delta}$ in the bundle, associated with operator $\Delta$. If we denote by $Q_{\Delta}$ the quantization, associated with pair $\left(\nabla^{\Delta}, \nabla_{c}\right)$ we get the following decomposition of the operator:

$$
\Delta=Q_{\Delta}(\sigma)+\sigma_{0}(\Delta),
$$

where $\sigma_{0}(\Delta) \in \operatorname{End}(\pi)$, is the subsymbol.

Moreover, this decomposition is natural in the sense that

$$
\begin{aligned}
Q_{A_{*}(\Delta)}\left(A_{*}(\sigma)\right) & =A_{*}\left(Q_{\Delta}(\sigma)\right), \\
\sigma_{0}\left(A_{*} \Delta\right) & =A_{*}\left(\sigma_{0}(\Delta)\right),
\end{aligned}
$$

for any automorphism $A$ of the bundle $\pi$.

Using this construction we introduce invariants of operator to be ArtinProcesi GL $(\pi)$-invariants for the set of operators $\left\{\sigma_{0}(\Delta), R_{k}(\sigma)\right\}$, where $k=$ $0,1, \ldots .,(n+2)(n-1) / 2$.

We call them the Artin-Procesi invariants of the operators.

Remark that they generate rational invariants of the $G$-action on the extended symbol space End $(\pi) \otimes \Sigma_{2} \oplus \operatorname{End}(\pi)$. Regular $G$-orbits in this space has codimension

$$
\nu_{0}=\nu+m^{2}
$$

and $\nu_{0}>n$ as well as $\nu>n$.

Therefore, due to the Rosenlicht theorem, there are $\nu_{0}$ algebraically independent Artin-Procesi invariants that generate the field of rational $G$-invariants on the extended symbol space.

\section{Equivalence of regular differential operators}

We say that a differential operator $\Delta \in \operatorname{Diff}_{2}(\pi, \pi)$ is regular at a point $p \in M$ if its symbol $\sigma \in \operatorname{End}(\pi) \otimes \Sigma_{2}(M)$ is regular at the point and among Artin-Procesi invariants $a_{1}, \ldots, a_{\nu_{0}}$ of the operator, defining the $G$-orbit of the pair $\left(\sigma(\Delta), \sigma_{0}(\Delta)\right)$, there are $n=\operatorname{dim} M$ invariants, say $a_{1}, . ., a_{n}$, such that differentials of functions $a_{1}(\Delta), . ., a_{n}(\Delta)$ are linear independent at the point $a \in M$.

Here $a_{i}(\Delta)$ are the values of invariants at the operator $\Delta$.

The functions $a_{i}(\Delta), i=1, . ., n$, define local coordinates in a neighborhood $U \ni p$. We call them $([5],[4], 6])$ natural coordinates.

Taking the values of the basic Artin-Procesi invariants $a_{1}, \ldots, a_{\nu_{0}}$ at $\Delta$ and express them in terms of the natural coordinates $a_{i}(\Delta), i=1, . ., n$, we get functions $F_{j}$, such that

$$
a_{j}(\Delta)=F_{j}\left(a_{1}(\Delta), . ., a_{n}(\Delta)\right), \quad n+1 \leq j \leq \nu_{0} .
$$

Remark that all rational $G$-invariants of the pair $\left(\sigma(\Delta), \sigma_{0}(\Delta)\right)$ are rational functions of the basic invariant and, due to (7), they are functions of natural coordinates in the neighborhood. 
Similar to ([4]) define natural local chart $\phi_{U}: U \rightarrow \mathbf{D}_{U} \subset \mathbb{R}^{n}$, where

$$
\phi_{U}(b)=\left(a_{1}(\Delta)(b), . ., a_{n}(\Delta)(b)\right),
$$

and let $F_{U}: \mathbf{D}_{U} \rightarrow \mathbb{R}^{\nu_{0}-n}$ be the function given by (7).

We call the triple $\left(\phi_{U}, \mathbf{D}_{U}, F_{U}\right)$ the model of $\Delta$ in $U$.

Theorem 5. Let differential operators $\Delta, \Delta^{\prime} \in \operatorname{Diff}_{2}(\pi, \pi)$ have the same model in the open set $U$.

Then there is an automorphism $A_{U} \in \mathrm{GL}\left(\pi_{U}\right)$ of the restriction of the bundle $\pi$ on domain $U$ such that $A_{U *}(\Delta)=\Delta^{\prime}$.

Proof. Condition that operators have the same model means that the pairs $\left(\sigma(\Delta), \sigma_{0}(\Delta)\right)$ and $\left(\sigma\left(\Delta^{\prime}\right), \sigma_{0}\left(\Delta^{\prime}\right)\right)$ belong to the same $G$-orbit at any point $a \in U$. Therefore, there exists the above automorphism $A_{U}$. This means that the quadratic forms $g_{\sigma}$ coincide in $U$ and we have $\nabla^{\prime}=A_{U *}(\nabla)$ for the associated connections. Therefore, $A_{U *}(\Delta)=\Delta^{\prime}$ in the neighborhood.

Corollary 6. Let differential operators $\Delta, \Delta^{\prime} \in \operatorname{Diff}_{2}(\pi, \pi)$ has models $\left(\phi_{U}, \mathbf{D}_{U}, F_{U}\right)$ and $\left(\phi_{U^{\prime}}, \mathbf{D}_{U^{\prime}}, F_{U^{\prime}}\right)$ in open sets $U \subset M$ and $U^{\prime} \subset M$ defined by the same basic invariants $a_{1}, . ., a_{n}$ and functions $F_{j}$.

Let $\widetilde{U} \subset U$ and $\widetilde{U^{\prime}} \subset U^{\prime}$ be open and simply connected domains such that $\phi_{U}(\widetilde{U})=\phi_{U^{\prime}}\left(\widetilde{U^{\prime}}\right) \subset \mathbf{D}_{U} \cap \mathbf{D}_{U^{\prime}}$.

Then there is an automorphism $A_{\widetilde{U}, \widetilde{U^{\prime}}}: \pi_{\widetilde{U}} \rightarrow \pi_{\widetilde{U^{\prime}}}$ such that $\left(A_{\widetilde{U}, \widetilde{U^{\prime}}}\right)_{*} \Delta=\Delta^{\prime}$.

Following $([4])$ we say that an atlas $\left\{\left(\phi_{U^{\alpha}}, \mathbf{D}_{U^{\alpha}}\right)\right\}$ given by models $\left\{\phi_{U^{\alpha}}, \mathbf{D}_{U^{\alpha}}, F_{U^{\alpha}}\right\}$ is natural if the sets of basic invariants $\left(a_{1}^{\alpha}, \ldots, a_{n}^{\alpha}\right)$ are different for different $\alpha$.

Let the operators $\Delta$ and $\Delta^{\prime}$ have the same natural atlas. Then isomorphisms $A_{U_{\alpha}}$ as well as $A_{U_{\alpha} \cap U_{\beta}}$ are defined up to multiplication by nonvanishing functions $f_{\alpha}$ and $f_{\alpha \beta}$. Therefore, the existence of the global isomorphism is equivalent to triviality of the cohomology class in $H^{1}\left(M, \mathbb{Z}_{2}\right)$, defining by the cocycle $\left\{f_{\alpha \beta}\right\}$. Summarizing we get the following.

Theorem 7. Two regular linear differential operators $\Delta, \Delta^{\prime} \in \operatorname{Diff}_{2}(\pi, \pi)$ on a manifold $M$, with $H^{1}\left(M, \mathbb{Z}_{2}\right)=0$, are Aut $(\pi)$-equivalent if and only if a natural atlas for operator $\Delta$ is the natural atlas for $\Delta^{\prime}$, i.e. if they have the same models.

\section{Acknowledgements}

The author was partially supported by the Russian Foundation for Basic Research (project 18-29-10013). 


\section{References}

[1] M. Artin, On Azumaya algebras and finite dimensional representations of rings, J. Algebra 11 (1969), 532-563.

[2] D. Husemoller, Fibre Bundles. Graduate Texts in Mathematics, New York: Springer, 1994.

[3] C. Procesi, The invariant theory of $n \times n$ matrices, Advances in Mathematics 19 (1976) 306-381.

[4] V. Lychagin, On geometrical structures, associated with linear differential operators of the 1st order, J. Geom. Phys. (2020).

[5] V. Lychagin, V. Yumaguzhin, Classification of the second order linear differential operators and differential equations, J. Geom. Phys. 130 (2018) $213-228$.

[6] V. Lychagin, V. Yumaguzhin, On structure of linear differential operators, acting on line bundles, J. Geom. Phys. 148 (2020) Article: 103549 (1-27).

[7] M. Rosenlicht, A remark on quotient spaces, An. Acad. Brasil. 35. (1963). 